\title{
A Brief Observational History of the Black-Hole Spacetimes
}

\author{
Wolfgang Kundt \\ Argelander Institute of Bonn University, Auf dem Hügel 71, 53121 Bonn, Germany \\ Correspondence should be addressed to Wolfgang Kundt; wkundt@astro.uni-bonn.de
}

Received 24 October 2014; Revised 17 December 2014; Accepted 18 December 2014

Academic Editor: Seyed H. Hendi

Copyright (C) 2015 Wolfgang Kundt. This is an open access article distributed under the Creative Commons Attribution License, which permits unrestricted use, distribution, and reproduction in any medium, provided the original work is properly cited.

In this year (2015), black holes (BHs) celebrate their 100th birthday, if their birth is taken to be triggered by a handwritten letter from Martin Schwarzschild to Albert Einstein, in connection with his newly found spherically symmetric vacuum solution.

\section{Introduction}

Black holes are brainchildren of Einstein-Hilbert's General Relativity Theory (GRT). They arose naturally as the nonsingular solutions describing the axially symmetric vacuum far fields of the gravitational collapse of a sufficiently massive (rotating) burnt-out star under its own weight and were subsequently likewise taken seriously on superstellar mass scales - at the centers of galaxies-as well as on distinctly substellar mass scales, as evaporating mountain-sized objects (in mass) formed during earlier cosmic epochs. The (more) general possibility of a (slightly) nonsymmetrical gravitational collapse without a regular event horizon-known under the name of "naked singularity" - was eliminated from consideration by Roger Penrose's postulate of "cosmic censorship" (CC, [1]), with the plausible expectation that collapsing bodies would always remove their nonfitting higher multipole moments via gravitational radiation-strongly suggested by the heroic work of Richard Price [2] - an expectation which has meanwhile been proven unjustified $[3,4]$ : $\mathrm{BH}$ spacetimes form a subset of measure zero within the class of all collapse scenarios.

Should we worry? I do not think so. Observations have shown that we live inside a comparatively young (part of the) Universe, with a significant fraction of its primordial hydrogen yet unburnt (towards chemical elements of higher nuclear binding energy). No $\mathrm{BH}$ has been reliably detected in our cosmic neighbourhood. Quite likely, all we have to do to save our regular physical description of this world is to replace Penrose's CC hypothesis by the slightly more restrictive one of AUC (avoidance of unhalted collapse).

\section{Birth and Growth of the Black Hole Idea}

It was the problem of unhalted gravitational collapse of large masses under their own weight, which emerged freshly with Einstein's GRT - in generalisation of Newton's Theorybecause, in GRT, pressure has weight (like mass or energy), and beyond certain (high) mass densities, pressures can no longer halt a collapse. The most massive neutron stars are close in density to the instability limit; they realise an extreme stellar population. What happens when additional matter is transferred to a heavy neutron star? This question opened the doors to $\mathrm{BH}$ favouritism.

This question is serious, but it is not fatal. Stars can lose mass through centrifugal ejection, through their winds, through their radiation, and through nuclear detonations, as novae-also through supernova explosions-and within the large set of known neutron stars, none so far is close in mass to its stability limit of $3 M_{\odot}$; they all have masses $\lesssim 2 M_{\odot}$. We realise that there are hurdles to $\mathrm{BH}$ formation.

$\mathrm{BH}$ research has not been easy, as it required, among others, explicit analytic solutions of the GRT equations and their complete analytic extensions from local to global ones; see the careful survey by Heusler [5] and also Kundt [6-9] and Thorne [10]. For a few years after their discovery, all of us worldwide were ready to believe that $\mathrm{BH}$ might turn out to be standard building blocks of the Universe. But it was equally clear to us, individually as well as in international discussion groups, for several years, that their detection in the sky would be far from easy. Because even their radiation cannot escape from them; they cannot emit signals, or blow winds, or (even) eject jets; they can only swallow, not spit, 
and only absorb, not emit. Their detections would have to be rather indirect, by carefully watching their surroundings at the right moments, at low signal strengths, because of escaping from near the bottom of a deep potential well. As concerns jets from BHs, supportive claims have been made in recent years, without proof and without reference to a proof, based only on imagination and speculation. Established jet engines are among the most sophisticated machines of the inorganic Universe; they involve strong, heavy rotating magnets and stable de Laval nozzles [11, 12]. For years, our best candidate for $\mathrm{BH}$ detection has been Cyg X-1, an Xray binary blowing jets intermittently containing a bright primary star and an unseen, heavy companion [13-16]: quite likely, the unseen companion is a neutron star surrounded by a massive accretion disk [17]. None of the rich classes of stellar-mass $\mathrm{BH}$ candidates has ultimately turned out to contain a BH [18]; our search had been in vain.

Another class of $\mathrm{BH}$ candidates was already proposed in [19] by Donald Lynden-Bell, through the fear that supermassive black holes, SMBHs, could form near the massive center of the deep potential well of a large galaxy. How can such accumulated matter be ejected again to large distances of lower gravity? A special conference at Bad Honnef in 1995, cochaired by Peter Scheuer, gave a tentative answer to this puzzle: the central galactic disks may well be supported by ordinary plasma pressure (perpendicular to disk plane) combined-in radial direction - with centrifugal forces, and their nuclear-burning matter may well be reejected into the CGM in the shape of fountains of galactic scale, observed as an active galaxy's burning disk (BD), broad-line region (BLR), NLR, ELR, and EER, out to $10^{2} \mathrm{Kpc}$ from its center and beyond, with the required power supplied by nuclear burning, in combination with conserved angular momentum from the past spiral-in motion through the disk [20]. This interpretation has meanwhile been corroborated by the SDSS plot of the core masses of $\leqslant 15000$ galaxies [21], whose masses decrease with cosmic time, from some $10^{9.5} M_{\odot}$ at $z \approx 4.5$, down to some $10^{6.5} M_{\odot}$ at present, as well as by the two halosized $\gamma$-ray lobes of our Milky Way mapped by the FERMI mission, which are fed in the vicinity of Sgr $\mathrm{A}^{*}$, the hard point source at our galactic center [12]. Already Victor Ambartsumian noted in [22] that galactic centers are observed to eject, rather than to swallow. And in [23] I argue that all the activities near Sgr $\mathrm{A}^{*}$ are satisfactorily described by a BD, whilst they are multiply inconsistent with a SMBH in its place.

\section{Black Hole Thermodynamics and the $(\geq 4)$ Classes of Black Holes}

Once the BH spacetimes had been mastered mathematically-culminating with Roy Kerr's metric for a rotating $\mathrm{BH}$ in 1963-and once the expected stellar-mass BHs and the likewise expected supermassive BHs in the galactic centers had been baptised-in 1971-by John A. Wheeler and Remo Ruffini in Princeton, backed up by Stephen Hawking et al. in England's Cambridge, it was a must to extend their considered mass range to the maximal physically expected one and to reflect on the specific properties of the subclasses: at this point, Stephen Hawking $[24,25]$ took the world's lead, by opening the chapter "BH Thermodynamics," at the seventh Texas Symposium on Relativistic Astrophysics in Dallas, a few days before Christmas 1974. He proposed a (massindependent) marriage of GR and quantum mechanics, by assigning a de Broglie wavelength $\lambda$ of the order of its horizon length to a $\mathrm{BH}$ of mass $M$ and with it a temperature $T:=\hbar c / \lambda k$, such that a $\mathrm{BH}$ of mass $M$ has a temperature $T(M) \approx 10^{-7} \mathrm{~K} M_{\odot} / M$. Obviously, this quantum temperature is ignorably small for $\mathrm{BHs}$ of stellar mass or bigger but could lead to detectable cosmic explosions for mountainsized BHs, when they shrink down by evaporation to the Hawking mass $M_{H}=\hbar c / G m_{\pi}=10^{15} \mathrm{~g}$ (with $m_{\pi}:=$ mass of the pion).

Instead of the hundreds of publications in this wide theoretical field, may I just list the names of a few of its leading authors. Beyond those already quoted, they are Brandon Carter, Werner Israel, Ted Newman, David Robinson, Jim Bardeen, Martin Rees, Jim Hartle, Jacob Bekenstein, and Robert Wald. Their considerations have led to a classification of all possible BHs into mini, midi, and maxi ones, each class ranging in mass through a factor of $\sqrt{\hbar c / G m_{\pi}^{2}} \approx 10^{20}$, starting at the bottom with the Planck mass $M_{\mathrm{Pl}}:=\sqrt{\hbar c / G}=10^{-5} \mathrm{~g}-$ whose Compton wavelength equals its Hawking wavelength - and extending up to the mass of the observable universe, $M_{U}=(\hbar c / G)^{2} / m_{\pi}^{3}=10^{55} \mathrm{~g}$. As concerns their detection, midi BHs have only been considered seriously once in 1974, as a possible explanation for the 1908 Tunguska catastrophe, even though no mechanism for their formation had ever been proposed. They were refuted by Beasley and Tinsley [26], based on an absence of tsunamis in the Pacific during the days of that event (which would have been raised by the midi $\mathrm{BH}$ during its exit from the ocean, after having crossed the Earth). Note that in my understanding, the Tunguska event has not been an infall event from outside, rather an ejection event from inside, a kimberlite [27].

Next, explosions of mini BHs have been ruled out by Joe Taylor by a large margin, via an absence of detected radio bursts of the implied kind. And I have never been shown convincing evidence of a maxi $\mathrm{BH}$ either, throughout the decades since their proposition [12]. Note that yet another class of BHs has been taken seriously in 2012, when CERN's Large Hadron Collider was assigned to search for the Higgs particle, quantum mini BHs, much lighter than the Planck mass, whose growth was feared to possibly swallow the whole city of Geneva. Fortunately for our home planet Earth, this most dangerous class of $\mathrm{BHs}$ has not shown up either.

$\mathrm{BHs}$ have thus remained unobserved objects in all weight classes. Even worse, the book by Yvan Leblanc [28] claims that $\mathrm{BH}$ thermodynamics is inconsistent with standard textbooks on physics. A number of further people support his view, among them Vladimir Belinski [29]. The publication of my own reply to Hawking's launching paper [24] on BH entropy, in [1976], was delayed by more than half a year and eventually printed without sending me proof sheets and with 13 typos added. In it, I pointed out that his definition of $\mathrm{BH}$ entropy was inconsistent with the textbook definition of entropy in physics. (Textbook entropy scales linearly with mass, 
Hawking's entropy scales quadratically with it.) Concerning proposals to combine GR with quantum mechanics, my own understanding is that quantum theory must not be applied to systems all of whose particles are purely macroscopic, that is, are much larger than their de Broglie wavelength. This criterion covers also the very recent proposal by Vaz [30].

\section{Should Cosmic Censorship Be Replaced by the AUC Hypothesis?}

Naked singularities were disliked from the beginning of $\mathrm{BH}$ history, in particular by Penrose [1], and have never appealed to astrophysicists, because their relevance would mean that we lack a complete theory of space and time. If future null geodesics could connect collapsing subsystems of the Universe with our telescopes, it would mean that these subsystems - or at least certain parts of them-can no longer be described by regular solutions of GRT, that is, that we lack a complete theory of our world. But the work of Pankaj Joshi [3], Hernando Quevedo, Bahram Mashhoon, Pankaj Joshi and Malafarina [4], and a few others has told us that the general gravitational collapse ends in naked singularities, rather than in BHs. What choice do we have?

So far, no spot in the sky has ever looked to me like containing a naked singularity. With sufficient care, all our maps have always allowed interpretations by nonsingular condensations of matter, not too dissimilar from our solar system. But clearly, if most of the cosmic hydrogen was replaced by iron, or just by carbon, similar considerations to the ones above would no longer predict hurdles to prevent unhalted collapse, on stellar as well as on superstellar mass scales. Burnt-out massive stars would collapse, and the central disks of galaxies would be squeezed out of equilibrium by their own weight, almost all of them forming naked singularities, hardly any of them forming a black hole. The fact that none of this has been observed so far makes me optimistic to believe that our cosmic neighbourhood is (still) far from this state, that we live at least in a stable subdomain of the Universe for which we can replace Roger's CC hypothesis by AUC, the hypothesis of an avoidance of unhalted collapse [27]. AUC postulates that in our presently observable cosmic neighbourhood, unhalted gravitational collapse does not happen.

Such an unhalted collapse can be avoided by the hurdles of nuclear burning, of conserved angular momentum, of stabilising ram pressures and/or magnetic pressures, and of jetlike ejections, all of which can delay a collapse. Star formation is delayed to (only) some $M_{\odot} / \mathrm{yr}$ in our present galaxy, stellar collapses are halted by nuclear burning or, during later stages, by degeneracy pressures inside compact stars, also by Nova and SN explosions, and galactic centers can likewise be supported against collapse by their pressures, by nuclear heating, and by explosive ejections from their central disks which delay their ultimate shrinkage until after the lifetime of our solar system.

Quite likely, we need not worry about the immediate future of terrestrial life. We had better concentrate on protecting our home planet Earth against the dangers of unhalted population growth, of nuclear catastrophes, of wastes from nuclear burning, and of plagues. They are far more to be feared than the ultimate death of distant stars and of distant galaxies in the Universe.

\section{Conflict of Interests}

The author declares that there is no conflict of interests regarding the publication of this paper.

\section{Acknowledgments}

My cordial thanks for the paper-including its contentsgo to Ole Marggraf and also to Hans Baumann. Thanks are likewise due to an anonymous referee who proposed five improvements of the original paper.

\section{References}

[1] R. Penrose, "Black holes," Scientific American, vol. 226, no. 5, pp. 38-46, 1972.

[2] R. H. Price, "Nonspherical perturbations of relativistic gravitational collapse. I. Scalar and gravitational perturbations," Physical Review. D. Particles and Fields, vol. 5, pp. 2419-2438, 1972.

[3] P. S. Joshi, "Naked singularities, the black hole has a troublesome sibling," Scientific American, February 2009, literally reprinted in vol. 22, no. 2, May 2014.

[4] P. S. Joshi and D. Malafarina, "Instability of black hole formation under small pressure perturbations," General Relativity and Gravitation, vol. 45, no. 2, pp. 305-317, 2013.

[5] M. Heusler, Black Hole Uniqueness Theorems, Cambridge Lecture Notes in Physics No. 6, Cambridge University Press, 1996.

[6] W. Kundt, "Global theory of spacetime," in Proceedings of the 13th Biennial Seminar of the Canadian Mathematical Congress (at Halifax, August 1971), J. R. Vanstone, Ed., vol. 1, pp. 93-133, Montreal, Canada, 1972.

[7] W. Kundt, "Schwarze Löcher," Physik in unserer Zeit, vol. 4, no. 6, pp. 181-186, 1973.

[8] W. Kundt, "Schwarze Löcher nach wie vor aktuell," Physik in Unserer Zeit, vol. 7, no. 5, pp. 131-135, 1976.

[9] W. Kundt, "Entropy production by black holes," Nature, vol. 259, no. 5538, pp. 30-31, 1976.

[10] K. S. Thorne, “The search for Black Holes," Scientific American, vol. 231, no. 6, 1974.

[11] W. Kundt, "Black holes cannot blow Jets," in Proceedings of the 12th Brazilian School of Cosmology and Gravitation: Mangaratiba, Novello and Bergliaffa, Eds., pp. 109-118, Cambridge Scientific Publishers, September 2011.

[12] W. Kundt, "Our Galactic center-the nearest burning disk," Acta Polytechnica, vol. 53, no. 1, pp. 506-511, 2013.

[13] J. N. Bahcall, F. J. Dyson, J. I. Katz, and B. Paczyński, “Multiple star systems and X-ray sources," The Astrophysical Journal, vol. 189, pp. L17-L18, 1974.

[14] A. C. Fabian, J. E. Pringle, and J. A. J. Whelan, "Is Cyg X-1 a neutron Star?” Nature, vol. 247, no. 5440, pp. 351-352, 1974.

[15] Y. Avni and J. N. Bahcall, "Ellipsoidal light variations and masses of X-ray binaries," The Astrophysical Journal, vol. 197, part 1, pp. $675-688,1975$. 
[16] A. W. Shafter, R. J. Harms, B. Margon, and J. I. Katz, "A lower limit on the magnitude of the companion to HDE 226868/ Cygnus X-1," The Astrophysical Journal, vol. 240, pp. 612-618, 1980.

[17] W. Kundt, "Cyg X-1-a neutron star surrounded by a massive disk?” Astronomy \& Astrophysics, vol. 80, no. 2, pp. L7-L10, 1979.

[18] W. Kundt and D. Fischer, "Is there a black hole among the blackhole candidates?" Journal of Astrophysics and Astronomy, vol. 10, no. 1, pp. 119-138, 1989.

[19] D. Lynden-Bell, "Galactic nuclei as collapsed old quasars," Nature, vol. 223, no. 5207, pp. 690-694, 1969.

[20] W. Kundt, Ed., Jets from Stars and Galactic Nuclei: Proceedings of a Workshop Held at Bad Honnef, Germany, 3-7 July 1995, vol. 471 of Lecture Notes in Physics, Springer, 1996.

[21] M. Vestergaard, X. Fan, C. A. Tremonti, P. S. Osmer, and G. T. Richards, "Mass functions of the active black holes in distant quasars from the sloan digital sky survey data release 3," The Astrophysical Journal Letters, vol. 674, no. 1, p. L1, 2008.

[22] V. Ambartsumian, "Report on AGN activities," in Proceedings of the Solvay Conference on Physics, Brussels, Belgium, 1958.

[23] W. Kundt, "Astrophysics without black holes, and without extragalactic gamma-ray bursts," Acta Polytechnica CTU Proceedings, vol. 1, no. 1, pp. 27-33, 2014.

[24] S. W. Hawking, "Black hole explosions?" Nature, vol. 248, no. 5443, pp. 30-31, 1974.

[25] S. W. Hawking, "Particle creation by black holes," Communications in Mathematical Physics, vol. 43, no. 3, pp. 199-220, 1975.

[26] W. H. Beasley and B. A. Tinsley, "Tunguska event was not caused by a black hole," Nature, vol. 250, no. 5467, pp. 555-559, 1974.

[27] W. Kundt and O. Marggraf, Physikalische Mythen auf dem Prüfstand, Springer, Berlin, Germany, 2014.

[28] Y. Leblanc, Quantum Black Holes: A Critical Analysis, eFieldTheory.COM, 2002.

[29] V. A. Belinski, "On the existence of black hole evaporation yet again," Physics Letters A, vol. 354, no. 4, pp. 249-257, 2006.

[30] C. Vaz, “There's nothing 'black' about a black hole," http://xxx .tau.ac.il/abs/1407.3823v1. 


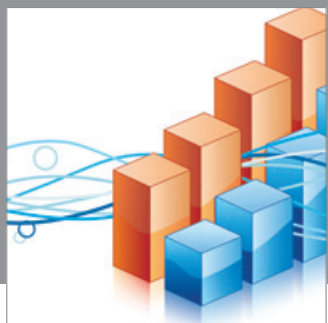

Advances in

Operations Research

mansans

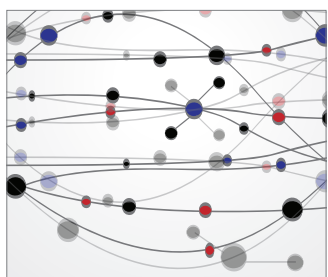

The Scientific World Journal
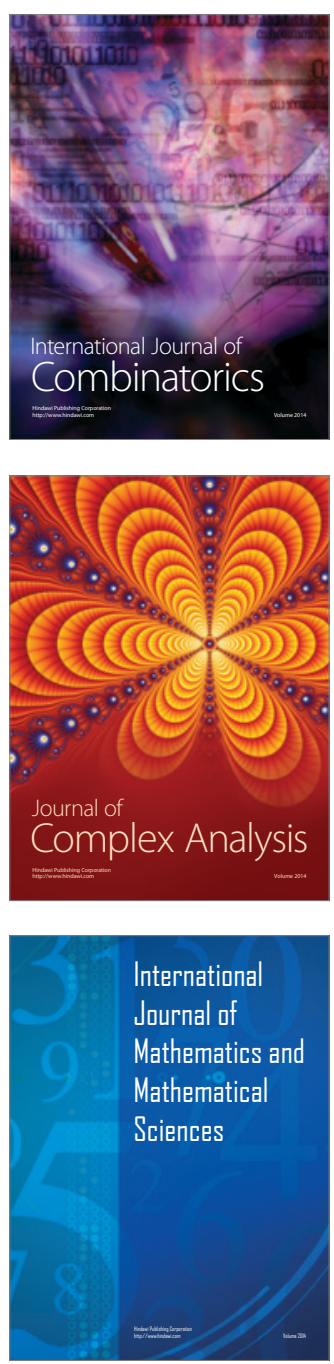
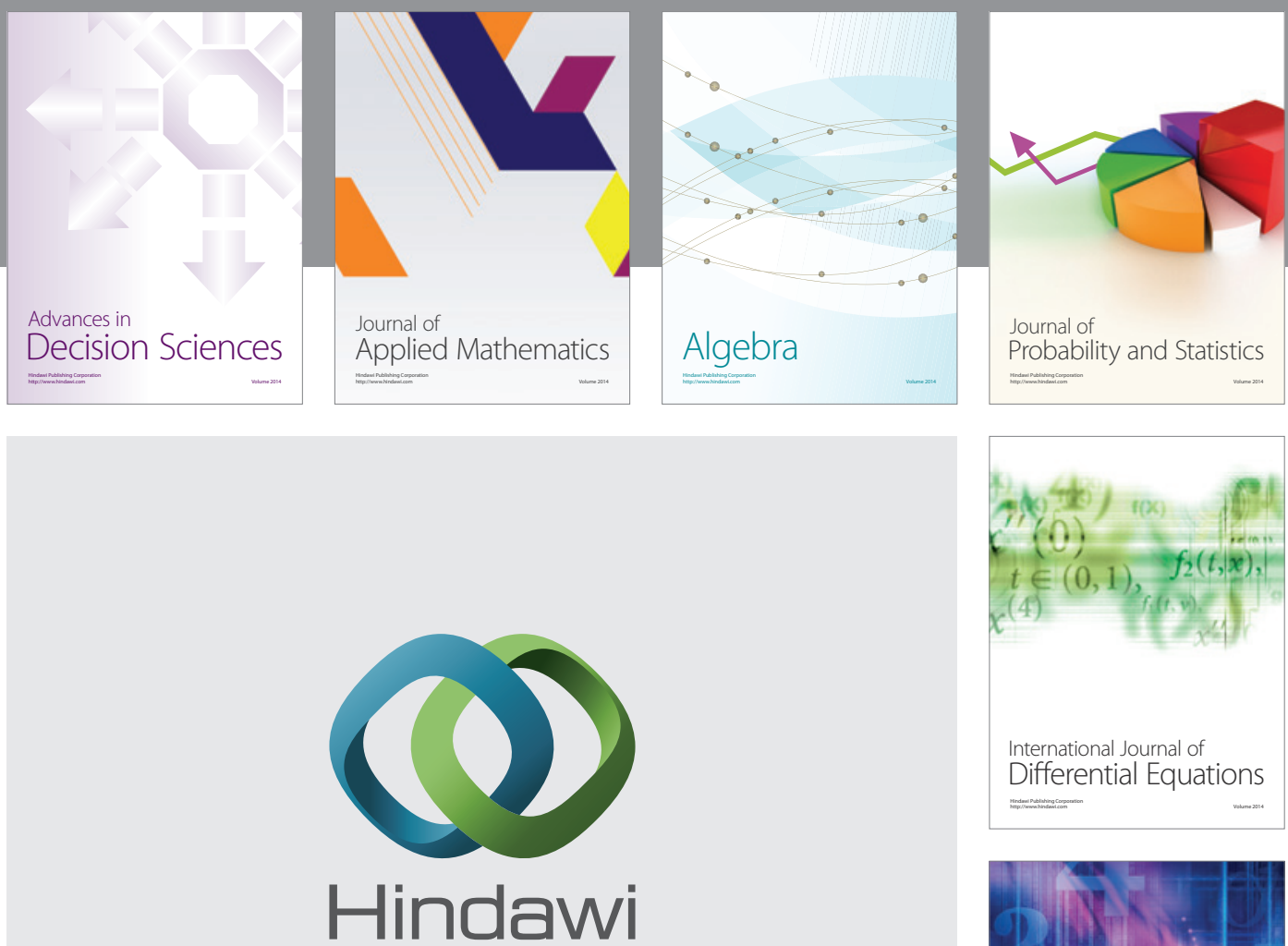

Submit your manuscripts at http://www.hindawi.com
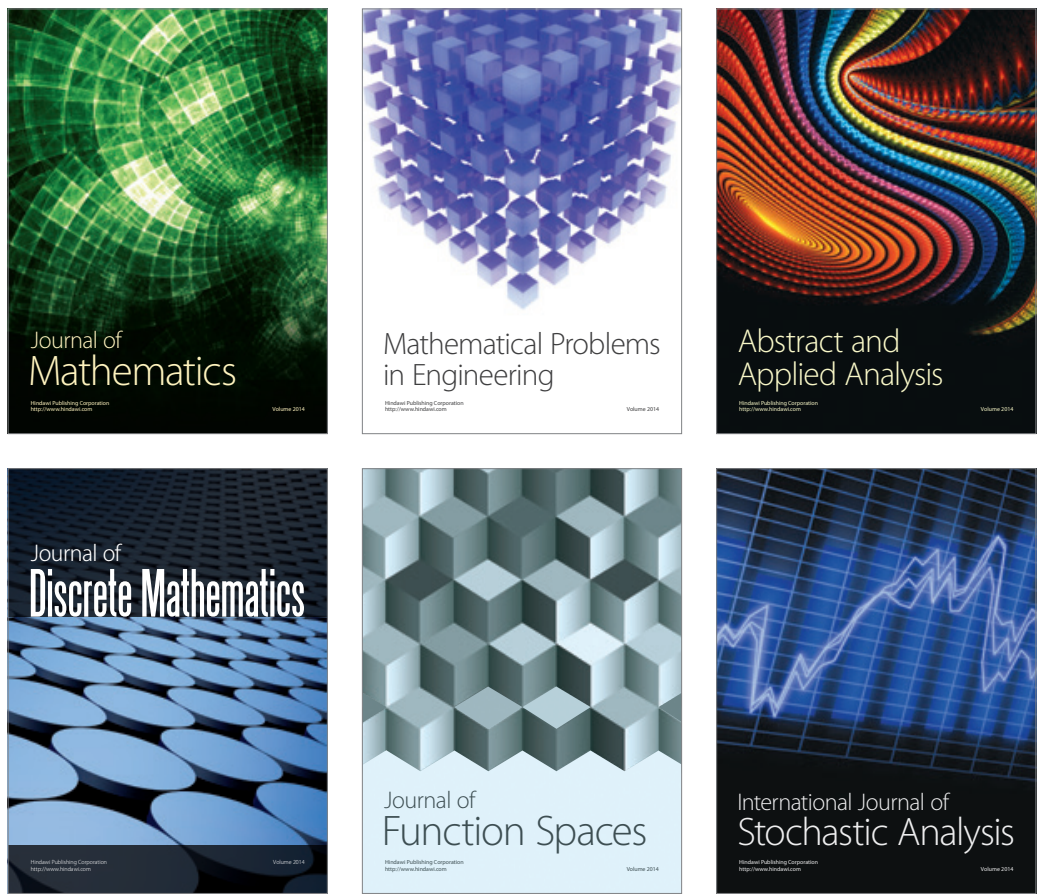

Journal of

Function Spaces

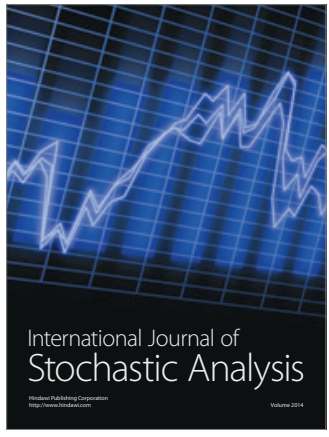

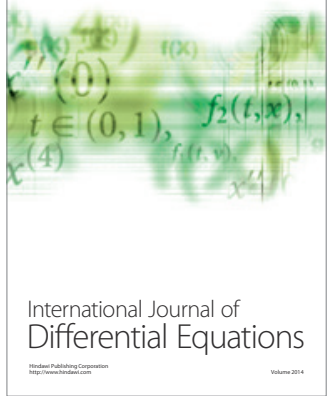
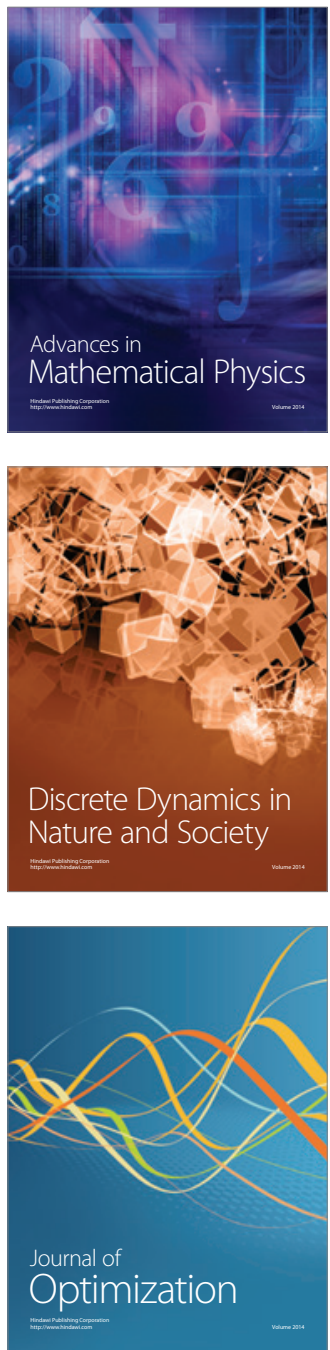